\title{
Picosecond Laser Study of Thioindigoid Dyes Photoisomerization
}

\author{
S. A. KRYSANOV and M. V. ALFIMOV \\ Institute of Chemical Physics, Academy of Sciences of the USSR, 142432 \\ Chernogolovka, Moscow Region, USSR
}

(Received 16 February 1983; in final form 10 May 1983)

The transient species in the trans $\rightleftharpoons$ cis photoisomerization of the perinaphto-thioindigoid dye were identified by picosecond laser spectroscopy in the time domain $10 \mathrm{ps}-5 \mathrm{~ns}$. An excitation of the trans isomer with a $6 \mathrm{ps}, 528 \mathrm{~nm}$ pulse to the singlet state $S_{1}$ (the peak of $S_{n} \leftarrow S_{1}$ absorption at $700 \mathrm{~nm}$ ) results in the intersystem crossing $S_{1} \leadsto T_{1}$ (the peak of $T_{n} \leftarrow T_{1}$ absorption at $730 \mathrm{~nm}$ ) with the quantum yield of 0.7 and rate constant $(1.4 \pm 0.3) \cdot 10^{9} \mathrm{~s}^{-1}$. An excitation of the cis isomer results also in the intersystem crossing (the peak of $T_{n} \leftarrow T_{1}$ absorption at $670 \mathrm{~nm}$ ) with the rate constant $(3.7 \pm 1.2) \cdot 10^{10} \mathrm{~s}^{-1}$. The triplet mechanism of the photoisomerization in the both directions is proposed.

\section{INTRODUCTION}

Thioindigoid dyes having a large difference in the trans and cis isomer absorption spectra are one of the most appropriate compounds for the reversible trans $\rightleftharpoons$ cis photoisomerization study. The common point of view on the mechanism of thioindigoid dyes photoisomerization is absent at present. An involvement of the trans isomer triplet state in the trans $\rightarrow$ cis photoisomerization was established ${ }^{1-4}$ by nanosecond absorption spectroscopy. The results of the fluorescence studies, ${ }^{5,6}$ were interpreted on the other hand in terms of photoisomerization in the singlet state of the trans isomer. The reverse cis $\rightarrow$ trans photoisomerization is the least studied and is the most interesting. According to the mechanism proposed in Refs. [7 and 8 ], the cis $\rightarrow$ trans photoisomerization occurs as a result of the transition from the lowest excited singlet state $S_{1}$ of the cis isomer into 
the state $S_{1}$ of the trans isomer. The trans singlet state $S_{1}$ can be depopulated to the ground $S_{0}$ state due to internal or intersystem crossing.

It is necessary to detect both the singlet and triplet states in the single experiment for the elucidation of the photoisomerization mechanism. The picosecond absorption spectroscopy was applied in the present work for this purpose.

\section{EXPERIMENTAL}

The formula of the perinaphtho-thioindigoid dye (PNT) under study is shown in Figure 1. Measurements on PNT in chloroform were carried out with undegassed solutions at $291 \mathrm{~K}$. The PNT cis isomers were prepared by illumination of trans solution with the $577 / 579 \mathrm{~nm}$ lines of a $1000 \mathrm{~W}$ mercury lamp selected by glass filters. Since the absorption spectra of the isomers of the PNT are well separated (Figure 1) it makes possible to convert the trans isomer completely into the cis isomer. The concentrations of the solutions were adjusted to give a ground-state optical density of 0.5 at the wavelength $528 \mathrm{~nm}$ in a $1 \mathrm{~mm}$ sample cell.

The apparatus used to measure transient absorbance spectra was the same as that described in detail elsewhere. ${ }^{9}$ Briefly, it includes a mode-locked Nd:phosphate glass laser on the basis of an unstable resonator configuration and amplifier system to produce single $\mathrm{TEM}_{00}$

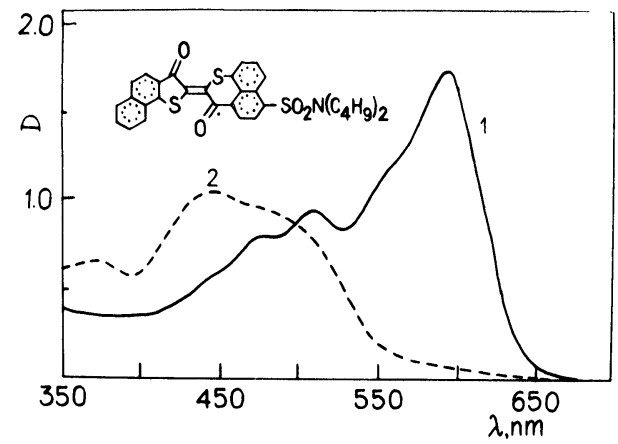

FIGURE 1 Ground-state absorption spectra of the pure trans (curve 1) and cis (curve 2) isomers of PNT dye in chloroform at room temperature. 
picosecond pulses at $1055 \mathrm{~nm}$. The second harmonic $528 \mathrm{~nm}$ with an energy of $0.5-1 \mathrm{~mJ}$ was used as an excitation pulse. For probing, the $1055 \mathrm{~nm}$ pulse generates in $\mathrm{D}_{2} \mathrm{O}$ a broad-band continuum. The double-beam optical configuration consisting of five miniature mirrors forms a number of the interrogating continuum pulses delayed by $10 \mathrm{ps}, 400 \mathrm{ps}, 1 \mathrm{~ns}, 2.5 \mathrm{~ns}$ and $5 \mathrm{~ns}$ relative to the excitation pulse. The absorbance changes $\Delta A$ in a sample are detected photographically and calculated for each delay time using two alternate laser shots with and without the excitation pulse. ${ }^{10}$ The angle between the polarization planes of excitation and probe beams was adjusted $55^{\circ}$ to eliminate effects due to rotational relaxation. ${ }^{11}$

The trans-PNT fluorescence lifetime was measured by the single photon counting with the subsequent deconvolution.

\section{RESULTS AND DISCUSSION}

On flashing a solution of trans-PNT in chloroform was the transient absorption band with the peak at $700 \mathrm{~nm}$ detected (Figure 2, curve 1). The transient is formed within the duration of the excitation pulse and its spectrum depends on the time. The displacement of the central maximum to $730 \mathrm{~nm}$ along with the formation of the shoulder at $660 \mathrm{~nm}$ and the new maximum at $820 \mathrm{~nm}$ are seen in the absorbance

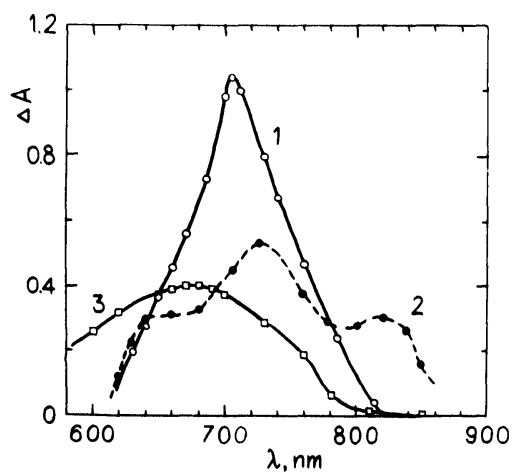

FIGURE 2 Transient absorbance spectra of trans-PNT measured at $10 \mathrm{ps}$ (1) and 5 ns delays (2) and of cis-PNT measured at 400 ps (3) delay after excitation with a $528 \mathrm{~nm}$ picosecond pulse. 
spectrum at the time moment 5 ns (Figure 2, curve 2). The kinetics of these spectral changes is shown in Figure 3, where the points with the zero absciss correspond to the 10 ps delay. Excitation of a sample with the $528 \mathrm{~nm}$ pulse results in the immediate bleaching (negative $\Delta A$ ) of the ground-state absorption band at $590 \mathrm{~nm}$. The transient absorption band $700 \mathrm{~nm}$ is formed simultaneously with the diminution of the $590 \mathrm{~nm}$ band. The optical density of the $700 \mathrm{~nm}$ band decays with the characteristic time of about 400 ps up to the some intermediate value corresponding to the long-lived induced absorption with the peak $730 \mathrm{~nm}$. The decay of the $700 \mathrm{~nm}$ band is accompanied in the same time interval by the partial recovery of the ground-state absorption band $590 \mathrm{~nm} . \Delta A$ at the wavelength of the cis isomer absorption has the negative sign and does not depend on time in the time interval 1-5 ns (Figure 3). These results define more exactly our previous data. ${ }^{12}$

As for the cis PNT, excitation by the picosecond pulse results in the formation of the broad induced absorption band (Figure 2, curve 3 ) with the maximum at about $670 \mathrm{~nm}$. The absorption band shape does not depend on time in the time interval $10 \mathrm{ps}-5 \mathrm{~ns}$. The absorption peak of the trans isomer at $590 \mathrm{~nm}$ was not detected in this time interval. It is worthwiile mentioning that this trans groundstate absorption absence when exciting the cis isomer can not be connected with the small yield of the trans isomer due to the small excitation pulse energy, for example. Indeed, we observed visually

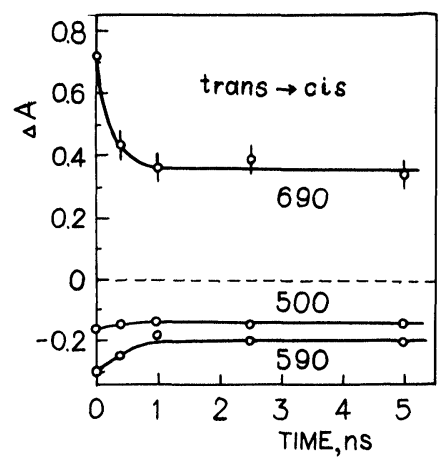

FIGURE 3 Absorbance kinetics at 690,500 and $590 \mathrm{~nm}$ of trans-PNT in chloroform at room temperature. Excitation with a $528 \mathrm{~nm}$ picosecond pulse. 
after the flash in the yellow solution of the cis isomer the presence of the strong violet spot of the trans isomer solution. The kinetic behavior of the transient from the cis isomer is shown in Figure 4. The buildup of transient absorption for cis-PNT following excitation at $528 \mathrm{~nm}$ was measured to be slower than that observed for trans PNT. The detailed study of the initial kinetic curve was carried out in Ref. 12 by means of the echelon and the risetime was shown to be $35 \pm 15$ ps. This feature of the slow going into the plateau is observed for all wavelengths (Figure 4) that proves the belonging of the induced absorption to the single transient species.

Let us consider the identity of the states which exhibit the transient absorption from the trans - and cis-PNT. Three states can be formed under excitation of the trans isomer: the lowest excited singlet $S_{1}$ (trans) and triplet $T_{1}$ (trans) and the ground state $S_{0}$ (cis). It is clear that the $S_{0}$ (cis) state can not be responsible for the $700 \mathrm{~nm}$ band since the cis absorption maximum is at $450 \mathrm{~nm}$ (Figure 1). The triplet state $T_{1}$ (trans) can not be also responsible for the $700 \mathrm{~nm}$ band since its risetime would be equal in this case to the fluorescence lifetime of the trans isomer $0,5 \pm 0.2 \mathrm{~ns}$. The only lowest excited singlet state $S_{1}$ of the trans isomer and not the two-step photodecomposition product can be responsible for the intense transient band $700 \mathrm{~nm}$. This assignment is supported by three facts. Firstly, its buildup is shorter than $10 \mathrm{ps}$; secondly, the decay time of the $700 \mathrm{~nm}$ band coincides with the fluorescence lifetime of the trans-PNT and thirdly,

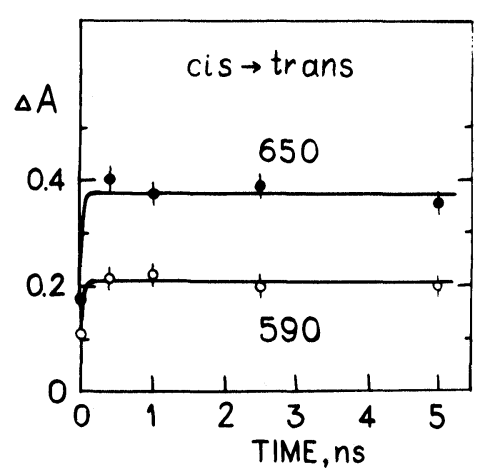

FIGURE 4 Absorbance kinetics at 650 and $590 \mathrm{~nm}$ of cis-PNT in chloroform at room temperature. Excitation with a $528 \mathrm{~nm}$ picosecond pulse. 
decay of the $700 \mathrm{~nm}$ band is accompanied by the recovery of the trans ground-state absorption. The observed intense band of the singlet-singlet absorption $S_{n} \leftarrow S_{1}$, in PNT at $700 \mathrm{~nm}$ locates also to the long-wavelength side of the $S_{1} \leftarrow S_{0}$ transition as in the case of thioindigo. ${ }^{13}$ Such location is probably the common property of the thioindigoid dyes. If the $700 \mathrm{~nm}$ band is assigned to the $S_{n} \leftarrow S_{1}$ absorption, it is quite naturally to assign the $730 \mathrm{~nm}$ band to the triplet-triplet absorption $T_{n} \leftarrow T_{1}$ of the trans-PNT. This assignment explains then the coincidence of the $730 \mathrm{~nm}$ band rise time with the $700 \mathrm{~nm}$ decay time and the long lifetime of the $730 \mathrm{~nm}$ band.

The only one transient with the $670 \mathrm{~nm}$ absorption band must be assigned in the case of the cis-PNT. According to model proposed in Ref. 8, cis-trans photoisomerization occurs via the triplet state of the trans form. It is necessary therefore to check in our experiment the possible population of four states: $S_{1}$ (cis), $S_{1}$ (trans), $T_{1}$ (trans) and $T_{1}$ (cis). The $670 \mathrm{~nm}$ band can not be assigned to states $S_{1}$ (trans) and $T_{1}$ (trans) since the absorption spectra from these states (curves 1 and 2, Figure 2) differ from that of the $670 \mathrm{~nm}$ transient. The $S_{1}$ (cis) state can not be responsible for the $670 \mathrm{~nm}$ band because of the slow buildup of the $670 \mathrm{~nm}$ band. We ascribe the $670 \mathrm{~nm}$ transient to the triplet-triplet absorption $T_{n} \leftarrow T_{1}$ of the cis-PNT. The intersystem crossing is responsible then for the slow buildup of about $35 \pm 15$ ps that corresponds to the rate constant $(3.7 \pm 1.2) \cdot 10^{10} \mathrm{~s}^{-1}$. These results indicate that excitation of the cis isomer results in the preferential population of the cis triplet state.

An analogous value of the intersystem crossing rate constant for the trans isomer can be obtained from the data presented in Figure 3. Moreover, the fraction $\alpha$ of the trans isomer converted into the cis isomer from the $S_{1}$ (trans) state can be evaluated from the Figure 3. The fact is that the stationary residual bleaching $\Delta A_{590}^{\text {st }}$ of the trans ground-state absorption at $590 \mathrm{~nm}$ is due to incomplete repopulation of the $S_{0}$ state from the $S_{1}$ state because of the photoisomerization (fraction $\alpha$ ) and intersystem crossing (fraction $1-\alpha$ ). Two species give a contribution to the stationary optical density $\Delta A_{500}^{s t}$ at $500 \mathrm{~nm}$, the bleached trans absorption and the formed cis absorption:

$$
\Delta A_{500}^{s t}=\left(\alpha \varepsilon_{500}^{c} / \varepsilon_{590}^{t}-\varepsilon_{500}^{t} / \varepsilon_{590}^{t}\right)\left|\Delta A_{590}^{s t}\right|=0.5(\alpha-1)\left|\Delta A_{590}^{s t}\right|
$$

where $\varepsilon=$ extinction coefficient, superscripts $t, c$ refer to the trans and $c i s$ isomers, subscripts - to the wavelength. The $\varepsilon$-ratios are 
readily obtained from the Figure $1 . A_{500}^{s t}=-0.10$ at $\alpha=0, \Delta A_{500}^{s t}=$ 0 at $\alpha=1$, according to the above relation. The experiment gives $\Delta A_{500}^{s t}=-0.14 \pm 0.03$ (Figure 3 ), that corresponds closely to the case $\alpha=0$. The intersystem crossing is thus responsible for the residual bleaching $\Delta A_{590}^{\text {st }}$. Its quantum yield $\varphi_{i s c}$ is equal to the ratio of the stationary and the initial $(t=10 \mathrm{ps})$ optical densities at $590 \mathrm{~nm}$. From the Figure 3 we obtain $\varphi_{\text {isc }}=0.70 \pm 0.15$. By means of the evident relationship $\varphi_{\text {isc }}=\tau_{f} \cdot K_{i s c}$ we obtain $K_{i s c}=(1.4 \pm 0.3) \cdot 10^{9-1} \mathrm{~s}$ taking into account the trans fluorescence lifetime $\tau_{f}=0.5 \mathrm{~ns}$.

The question that arises is the reason for the large difference between the trans and cis intersystem crossing rates. The mechanism we propose is shown in Figure 5. Our conclusion about the triplet mechanism of the trans $\rightarrow$ cis photoisomerization is in agreement with that of the previous nanosecond experiments. ${ }^{1-4,7,8}$ There is a large potential barrier in the trans $S_{1}$ state that prevents the rotation in the singlet state. The cis isomer has on the contrary no such barrier in its $S_{1}$ state, and the fast rotation in the $S_{1}$ state is the primary process upon excitation of the cis isomer. Such rotation results in the decrease of the $S_{1}-T_{1}$ energy gap and in the increase of the spin-orbit coupling. Both possible phenomena increase drastically the intersystem crossing rate already on the initial stage of the rotation. ${ }^{14-16}$ This picture of the preferential cis intersystem crossing differs somewhat from that of the model of Memming et al. ${ }^{7,8}$ where the possibility of the large rotation angles in the $S_{1}$ (cis) state is assumed.

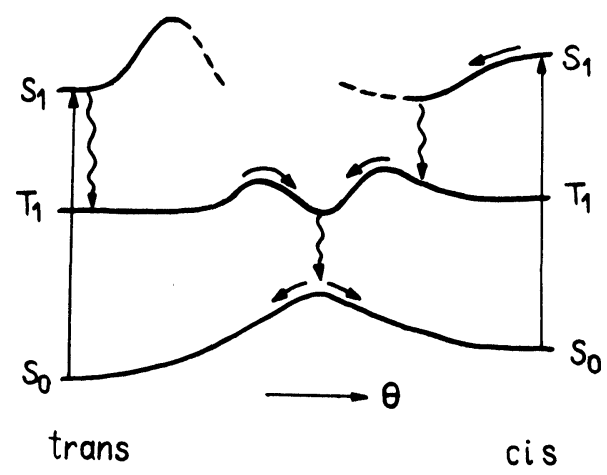

FIGURE 5 The proposed scheme of trans $\rightleftharpoons$ cis photoisomerization of thioindigoid dyes. 


\section{Acknowledgement}

We gratefully acknowledge Dr. Zoya A. Chizhikova and Prof. Mikhail D. Galanin for the recording of the trans-PNT fluorescence by a single photon counting and Dr. Georgy V. Maier for helpful discussion.

\section{References}

1. D. Schulte-Frohlinde, H. Herrmann and G. M. Wyman, Z. Physik. Chem. 101, 115 (1976).

2. H. Görner and D. Schulte-Frohlinde, Chem. Phys. Lett. 66, 363 (1979).

3. K. H. Grellman and P. Hentzschel, Chem. Phys. Lett. 53, 545 (1978).

4. T. Karstens, K. Kobs and R. Memming, Ber. Bunsenges. Phys. Chem. 83, 504 (1979).

5. G. Haucke and R. Paetzold, J. prakt. Chem. 321, 978 (1979).

6. T. Karstens, Ber. Bunsenges. Phys. Chem. 86, 315 (1982).

7. R. Memming and K. Kobs, Ber. Bunsenges. Phys. Chem. 85, 238 (1981).

8. C. P. Klages, K. Kobs and R. Memming, Chem. Phys. Lett. 90, 46 (1.982).

9. S. A. Krysanov and M. V. Alfimov, Chem. Phys. Lett. 76, 221 (1980); 98, 176 (1983).

10. D. Huppert, P. M. Rentzepis and D. S. Kliger, Photochem. Photobid. 25, 193 (1977).

11. H. E. Lessing and A. von Jena, Chem. Phys. Lett. 42, 213 (1976).

12. S. A. Krysanov and M. V. Alfimov, Chem. Phys. Lett. 82, 51 (1981).

13. S. A. Krysanov and M. V. Alfimov, J. Photochem. in press.

14. V. G. Plotnikov and G. V. Maier, Opt. i Spectr. 47, 113 (1979).

15. G. V. Maier and V. G. Plotnikov, Opt. i Spectr. 47, 310 (1979).

16. V. G. Plotnikov, Uspekhi khimii 49, 327 (1980). 